\title{
Prevention of congenital Chagas through treatment of girls and women of childbearing age
}

\author{
Guillermo Moscatelli/', Samanta Moroni, Facundo García-Bournissen, \\ Griselda Ballering, Margarita Bisio, Héctor Freilij, Jaime Altcheh
}

Department of Parasitology and Chagas, Ricardo Gutiérrez Children's Hospital, Buenos Aires, Argentina

It is currently unknown whether treatment of Chagas disease decreases the risk of congenital transmission from previously treated mothers to their infants. In a cohort of women with Chagas disease previously treated with benznidazole, no congenital transmission of the disease was observed in their newborns. This finding provides support for the treatment of Chagas disease as early as possible.

Key words: Trypanosoma cruzi - Chagas disease - congenital transmission - prevention - treatment

Chagas disease is a zoonotic disease caused by the Trypanosoma cruzi parasite endemic to the Americas. Most infections occur in children by vector or congenital transmission. Other routes of infection such as blood transfusion, organ transplants and oral transmission are less frequent. In areas where vector transmission is under control, such as Argentina, congenital transmission has become the most common route of transmission of the disease (Moscatelli et al. 2013).

The prevalence of Chagas disease in pregnant women in Latin America ranges from 5-40\% depending on the geographical area and the rate of congenital transmission is estimated to be $4-10 \%$. An infected mother can have several infected children, as vertical transmission can take place in either the acute or chronic stage of infection (Altcheh 2010, Moscatelli et al. 2013). The Pan American Health Organization estimates that there are currently 1,600,000 infected people in Argentina and approximately 1,000 new congenital infections occur every year (Altcheh 2010, Moscatelli et al. 2013).

The etiological treatment of Chagas disease with benznidazole (BZ) or nifurtimox (NFX) has proven to be effective in the paediatric population, both during the acute and chronic stages of infection (Freilij \& Altcheh 1995).

Drug treatment during pregnancy is not currently recommended due to the lack of foetal safety data for currently available drugs (PAHO 1999). Management of congenital infections requires universal screening of newborns from infected mothers to identify infected babies for whom treatment is highly effective. Taking into account that there is evidence of treatment effectiveness in children and adults (Viotti et al. 2006), treating infected girls and women of childbearing age has the potential to be an effective measure to prevent congenital transmission in future pregnancies.

doi: $10.1590 / 0074-02760140347$

+ Corresponding author: gfmoscatelli@yahoo.com.ar

Received 22 September 2014

Accepted 25 February 2015
The objective of this study was to follow a cohort of girls and women, previously treated for Chagas disease, to assess the risk of congenital transmission to their newborn babies.

\section{PATIENTS, MATERIALS AND METHODS}

Study design - Patients included in this study were selected from a cohort study of female patients assisted in the Parasitology and Chagas service of the Ricardo Gutiérrez Children's Hospital in Buenos Aires, Argentina, between 2003-2013. According to the local protocol, patients were treated with BZ or NFX. Inclusion criteria: pregnant women from our patient cohort treated for Chagas disease in childhood or early adulthood who brought their children in for evaluation for potential congenital transmission of Chagas disease were included in this study. All of the women resided in areas with no vector transmission after etiological treatment. These patients lived in the city of Buenos Aires, an area that has no reports of vector transmission.

The following data were gathered from clinical charts: demographic background, T. cruzi specific serology (pre-treatment and regularly for up to three years post-treatment), pre and post-treatment real-time quantitative polymerase chain reaction (qPCR), clinical course and clinical laboratory information. Serology methods used for diagnosis and follow up were indirect haemagglutination (IHA) (Polychaco Laboratory, Argentina) and ELISA (Wiener Laboratory, Argentina). An IHA titre $\geq$ $1 / 16$ and ELISA $R \geq 1.1$ were considered to be positive.

qPCR assays were performed as described by Duffy et al. (2013). Briefly, blood samples treated with guanidine ethylenediamine tetraacetic acid buffer were processed. A multiplex qPCR assay able to quantify nuclear T. cruzi and internal positive control DNAs was carried out using primers (cruzi1, cruzi2, IACTq Fw, IACTq $\mathrm{Rv}$ ) and TaqMan probes (cruzi3-FAM and IACTq-VIC) previously described (Duffy et al. 2013). All children underwent evaluation to exclude congenital infection. Parasitaemia was measured by the microhaematocrit method in infants under eight months old and serology for children and infants older than eight months old. Infants were considered not to have congenital infection when serology was negative after eight months of life. 
This study was approved by the Research Committee and the Ethical Review Board, Buenos Aires Children's Hospital Ricardo Gutiérrez.

\section{RESULTS}

In a cohort of 394 female patients treated in our service, 15 women had pregnancies (16 children) and were included in this study.

Information about the mothers - Average age at the beginning of treatment was 17 years old (between 9-34 years old). Mean time between treatment and birth of the children was five years (from 1-8 years). Place of birth: $10(66.7 \%)$ in Argentina and five (33.3\%) in Bolivia. Nine $(60 \%)$ lived in an endemic area. Infection route was not determined in six patients $(40 \%)$, congenital in six patients $(40 \%)$ and vector transmission in three patients (20\%). The mothers of $14 / 15(93.3 \%)$ women in the study were also affected by Chagas disease.

In $14 / 15$ patients ( $93.3 \%$ ) BZ treatment was prescribed, with a median dose of $6.2 \mathrm{mg} / \mathrm{kg} /$ day b.i.d. (range of 5-7.6 $\mathrm{mg} / \mathrm{kg} /$ day). One patient (6.6\%) received $9.4 \mathrm{mg} / \mathrm{kg} / \mathrm{day}$ of NFX. Mean duration of treatment was 51 days (1960 days). No patients had serious adverse drug events (ADR) during treatment. Only two/15 (13.3\%) presented mild ADR (exanthema in body and extremities and headache, in both patients). Before treatment, all mothers presented reactive serology measured by IHA and ELISA. During post-treatment follow-up, a 34\% decrease in antibody titre was observed as measured by ELISA and a $30.5 \%$ decrease was measured by IHA compared to the baseline value (i.e., when diagnosis was done). Negative seroconversion was observed in one patient (6.7\%). The mother that showed negative seroconversion had been treated at the age of nine years old. qPCR before treatment was positive in $13 / 15$ patients $(86.7 \%)$ and negative by the end of treatment in all patients (Table).

Infant outcomes - Sixteen children were included in this study (1 mother gave birth to 2 children after the treatment). All of the children were full term infants of appropriate weight for their gestational age and had no perinatal infectious diseases or complications. Parasitaemia, evaluated by the microhaematocrit method during the first days of life, was negative in all cases. All infants were re-evaluated at eight months of age by ELISA and IHA serology, which was negative in all cases, ruling out congenital infection.

\section{DISCUSSION}

Currently available treatment options for $T$. cruzi infection are BZ and NFX, which have been proven to be effective during the acute stage of the disease. Recent publications reinforce their prescription during the chronic stage of the disease (Viotti et al. 2006, Molina et al. 2014).

Negativisation of parasitaemia tested by qPCR, together with a lack of antibodies, has been proposed by some authors as criteria for parasitological cure (Altcheh et al. 2014). Our results in patients who received etiological treatment show a decrease in antibody titres after treatment and negative seroconversion in one patient, which is an indication of a complete response. This reinforces the benefits of etiological treatment in girls and women of childbearing age to prevent congenital transmission (Hermann et al. 2004).

TABLE

Serology and mother real-time quantitative polymerase chain reaction (qPCR) results throughout follow-up before treatment and three years after treatment - Children study

\begin{tabular}{|c|c|c|c|c|c|c|}
\hline \multirow{2}{*}{$\begin{array}{l}\text { Mothers } \\
\text { [age at treatment (years)] }\end{array}$} & \multicolumn{2}{|c|}{$\begin{array}{l}\text { Serology } \\
\text { (day 0) }\end{array}$} & \multicolumn{2}{|c|}{$\begin{array}{l}\text { Serology } \\
\text { (3 years) }\end{array}$} & \multicolumn{2}{|c|}{ qPCR } \\
\hline & EIA & IHA & EIA & IHA & Day 0 & 3 years \\
\hline 9 & 3.5 & 256 & UN & UN & $\mathrm{P}$ & $\mathrm{N}$ \\
\hline 11 & 10.6 & 64 & 4.1 & 64 & $\mathrm{P}$ & $\mathrm{N}$ \\
\hline 11 & 6.4 & 64 & 6.7 & 64 & $\mathrm{P}$ & $\mathrm{N}$ \\
\hline 12 & 8.9 & 256 & 6.2 & 512 & $\mathrm{P}$ & $\mathrm{N}$ \\
\hline 13 & 11 & 128 & 2.8 & UN & $\mathrm{P}$ & $\mathrm{N}$ \\
\hline 13 & 10.5 & 256 & 5.4 & 16 & $\mathrm{P}$ & $\mathrm{N}$ \\
\hline 14 & 8.1 & 128 & 4.3 & 16 & $\mathrm{~N}$ & $\mathrm{~N}$ \\
\hline 14 & 7.4 & 64 & 5.1 & UN & $\mathrm{P}$ & $\mathrm{N}$ \\
\hline 14 & 3.1 & 16 & 2.5 & 32 & $\mathrm{~N}$ & $\mathrm{~N}$ \\
\hline 16 & 11.7 & 1,024 & 11.3 & 1,024 & $\mathrm{P}$ & $\mathrm{N}$ \\
\hline 20 & 12.4 & 1,024 & 7.2 & 64 & $\mathrm{P}$ & $\mathrm{N}$ \\
\hline 20 & 10.6 & 128 & 6.6 & 64 & $\mathrm{P}$ & $\mathrm{N}$ \\
\hline 21 & 12 & 512 & 10.2 & 128 & $\mathrm{P}$ & $\mathrm{N}$ \\
\hline 29 & 11.6 & 1,024 & 6.6 & 256 & $\mathrm{P}$ & $\mathrm{N}$ \\
\hline 34 & 12.1 & 2,048 & 9.5 & 128 & $\mathrm{P}$ & $\mathrm{N}$ \\
\hline
\end{tabular}

EIA: enzymatic immunoassay; IHA: indirect haemagglutination assay; N: negative; P: positive; UN: unreactive. 
The main limitations in testing treatment responses through conventional serology during the chronic stage arise from the need for long-term follow-up (years or decades) in order to observe negative seroconversion of conventional serology tests. We have proven, in addition to other authors (Viotti et al. 2006), that patients treated during the chronic stage of $T$. cruzi infection show a gradual decrease in antibodies. In some cases, negative seroconversion is observed after several years, which is an indication of a complete response (Altcheh et al. 2005).

A new perspective on the evaluation of therapeutic response is based on the use of qPCR (Molina et al. 2014). Our results show negative seroconversion and qPCR of a mother treated when she was nine years old. In the rest of treated mothers in our study, the sustained decrease, throughout follow-up of serology titres measured by IHA and ELISA, together with negative qPCR results after treatment suggest that qPCR is a good therapeutic response marker, as we have seen in a cohort of treated children (Altcheh et al. 2011). We believe that studies should be performed on larger numbers of adult patients to validate these findings. Our patients did not show positive qPCR results after treatment, which would suggest therapeutic failure.

There is no evidence proving the effectiveness of treatment during pregnancy to prevent transmission. However, we have published the case of an human immunodeficiency virus reactive pregnant patient with a brain mass due to $T$. cruzi reactivation, treated with BZ at the 26th week of gestation with excellent responses and no evidence of ADRs either in the mother or the baby. Interestingly, vertical transmission of $T$. cruzi infection was not observed in the baby, born several weeks later (Bisio et al. 2013). We believe that more detailed studies are necessary to validate these findings.

The congenital infection rate observed in our region is estimated at $4-10 \%$ (Moscatelli et al. 2013). In our study, we did not detect any cases of vertical transmission among the offspring of the mothers treated before pregnancy. Although the number of mothers treated in our study is not high, our findings reinforce previously published data suggesting the benefits of etiological treatment in women of childbearing age to prevent congenital transmission (Altcheh et al. 2005). We believe that a larger sample size is needed to obtain a definitive conclusion, as well as a larger group of untreated mothers, in order to compare the effect of intervention to prevent congenital transmission.

Due to the migratory phenomenon that has occurred in past decades, Chagas disease has changed its epidemiological profile, becoming predominantly urban. Although monitoring of blood banks and vector transmission control are essential tools to manage T. cruzi infection, interruption of congenital transmission is a critical step that needs to be solved in order to prevent further disease transmission.

Prescribing treatment to infected women of childbearing age not only has the potential to reduce morbid- ity and mortality caused by heart disease and digestive disorders in those patients, but it can also be an effective strategy to prevent congenital transmission of T. cruzi, the main route of transmission of Chagas disease in $\mathrm{Ar}-$ gentina and in areas with no vector transmission.

\section{REFERENCES}

Altcheh J 2010. La enfermedad de Chagas a 100 años de su descubrimiento. Arch Argent Pediatr 108: 4-5.

Altcheh J, Biancardi M, Lapeña A, Ballering G, Freilij H 2005. Enfermedad de Chagas congénita, experiencia del Hospital de Niños Dr Ricardo Gutiérrez, Buenos Aires. Rev Soc Bras Med Trop 38: 41-45.

Altcheh J, Bisio M, Moscatelli G, Moroni S, García-Bournissen F, Schijman A, Ballering G, Ribeiro I 2014. PCR as a marker of cure in pediatric Chagas disease. Proceedings of the 32nd Annual Meeting of the European Society for Pediatric Infectious Diseases, 2014 May 6-10, Dublin, Abstract 98. Available from: espid2015.kenes.com/PublishingImages/scientific-information/ espid-abstracts/ESPID\%202014\%20abstracts.pdf.

Altcheh J, Moscatelli G, Moroni S, García-Bournissen F, Freilij H 2011. Adverse events after the use of benznidazole in infants and children with Chagas disease. Pediatrics 127: e212-218.

Bisio M, Altcheh J, Lattner J, Moscatelli G, Fink V, Burgos J, GarcíaBournissen F, Schijman A, Freilij H 2013. Benznidazole treatment of chagasic encephalitis in pregnant woman with AIDS. Emerg Infect Dis 19: 1490-1492.

Duffy T, Cura CI, Ramirez JC, Abate T, Cayo NM, Parrado R, Bello ZD, Velazquez E, Muñoz-Calderón A, Juiz NA, Basile J, Garcia L, Riarte A, Nasser JR, Ocampo SB, Yadon ZE, Torrico F, de Noya BA, Ribeiro I, Schijman AG 2013. Analytical performance of a multiplex real-time PCR assay using TaqMan probes for quantification of Trypanosoma cruzi satellite DNA in blood samples. PLoS Negl Trop Dis 37: e2000.

Freilij H, Altcheh J 1995. Congenital Chagas disease: diagnostic and clinical aspects. Clin Infect Dis 21: 551-555.

Hermann E, Truyens C, Alonso-Vega C, Rodriguez P, Berthe A, Torrico F, Carlier Y 2004. Congenital transmission of Trypanosoma cruzi is associated with maternal enhanced parasitemia and decreased production of interferon-gamma in response to parasite antigens. J Infect Dis 189: 1274-1281.

Molina I, Gómez i Prat J, Salvador F, Treviño B, Sulleiro E, Serre N, Pou D, Roure S, Cabezos J, Valerio L, Blanco-Grau A, SánchezMontalvá A, Vidal X, Pahissa A 2014. Randomized trial of posaconazole and benznidazole for chronic Chagas disease. $N$ Engl $J$ Med 370: 1899-1908.

Moscatelli G, García-Bournissen F, Freilij H, Berenstein A, Tarlovsky A, Moroni S, Ballering G, Biancardi M, Siniawski S, Schwarrcz M, Hernández S, Espejo-Cozzi A, Altcheh J 2013. Impact of migration on the occurrence of new cases of Chagas disease in Buenos Aires city, Argentina. J Infect Dev Ctries 157: 635-637.

PAHO - Pan-American Health Organization 1999. Etiological treatment for Chagas disease: conclusions of a technical consultation, OPS/HCP/HCT/140/99 2008, Washington, 34 pp.

Viotti R, Vigliano C, Lococo B, Bertocchi G, Petti M, Alvarez MG, Postan M, Armenti A 2006. Long term cardiac outcomes in treating chronic Chagas disease with benznidazole versus no treatment: a nonrandomized trial. Ann Intern Med 144: 724-734. 